\title{
Transport equation for the two-dimensional electron liquid under microwave radiation and a magnetic field: Explanation for the zero-resistance state
}

\author{
Tai-Kai $\mathrm{Ng}$ and Lixin Dai \\ Department of Physics, Hong Kong University of Science and Technology, Clear Water Bay Road, Hong Kong
}

(Received 11 August 2005; revised manuscript received 4 October 2005; published 29 December 2005)

\begin{abstract}
A general transport equation for the center of mass motion is constructed to study transports of an electronic system under uniform magnetic field and microwave radiation. The equation is applied to study the twodimensional (2D) electron system in the limit of weak disorder where negative resistance instability is observed when the radiation field is strong enough. A solution of the transport equation with spontaneous ac is proposed to explain the experimentally observed radiation-induced zero-resistance state.
\end{abstract}

DOI: 10.1103/PhysRevB.72.235333

PACS number(s): 73.40.-c, 73.50.-h, 78.67.-n

The discovery of the zero-resistance states (ZRS) in two dimensional electron gas under uniform magnetic field ${ }^{1,2}$ and microwave radiation has triggered a lot of theoretical ${ }^{3-9,12}$ and experimental activities ${ }^{10,11}$ to understand the origin of this nontrivial state. Most of the theoretical work suggests that the origin of the ZRS is closely related to a negativeresistance instability that occurs in the system due to the combined effect of quantized Landau levels and photonassisted scattering. ${ }^{3-7,12}$ It was proposed that the ZRS can be explained if the current-dependent resistance of the system which becomes negative at small current (for strong enough microwave radiation) becomes positive again when the current $\vec{j}$ becomes large enough. ${ }^{3-7}$ The above physics was put together phenomenologically into an equation

$$
\vec{E}_{d}=\rho_{H}[\vec{j} \times \hat{z}]+R[|\vec{j}|] \vec{j}
$$

where $R[j]$ is a phenomenological current-dependent resistance which is negative at $j=0$, increases as a function of $j$ and passes through zero at $|\vec{j}|=j_{o},{ }^{7} \vec{E}_{d}$ is the applied dc electric field and $\rho_{H}$ is the ordinary Hall resistivity. It was suggested that Eq. (1) admits a time-independent, stripelike spatially inhomogeneous solution which leads to zero differential resistance for net dc current less than a threshold value. $^{7}$ An obvious theoretical question is whether Eq. (1) with the required property of $R[j]$ can be derived microscopically. This is the subject of this paper.

Starting from first principles we shall derive in the following a transport equation for the center of mass velocity $\vec{v}$ $=\vec{j} /$ ne that treats the effect of radiation to all order with the only expansion parameter in the problem being the strength of disorder. Our approach is very similar to the approach adopted by Lei et $a l .{ }^{4}$ although the final result is different because of the different approximations involved. We note that a transport equation can also be derived from a quantum Boltzmann equation approach. ${ }^{12}$ However, it is difficult to obtain a clear analytical result in this approach because of the intrinsic complexity of the Boltzmann equation formulation itself and the equation of motion for the center of mass offers a much simpler alternative. ${ }^{4}$ We find that the equation of motion we derive differs from Eq. (1) in one important as- pect which leads to an alternative solution which can also explain the ZRS.

\section{DERIVATION OF THE TRANSPORT EQUATION}

Our approach to the transport equation begins from the known observation that there exists an exact, one-to-one mapping between the solution of the Schrödinger equation of a (charged) many-particle system in the absence of the microwave radiation and in the presence of the radiation for a class of Hamiltonian, $4,8,9,12,13$

$$
H(t)=H_{o}-e \sum_{i} \vec{r}_{i} \cdot \vec{E}(t),
$$

where

$$
\begin{aligned}
H_{o}\left(\vec{r}_{i}\right)= & \sum_{i} \frac{1}{2 m}\left(\vec{p}_{i}-\frac{e}{c} \vec{A}\left(\vec{r}_{i}\right)\right)^{2}+\frac{1}{2} \sum_{i \neq j} V\left(\vec{r}_{i}-\vec{r}_{j}\right) \\
& +\frac{1}{2} \sum_{i} \vec{r}_{i} \cdot \stackrel{\leftrightarrow}{K} \cdot \vec{r}_{i},
\end{aligned}
$$

where $\vec{p}_{i}=-i \bar{h} \nabla_{i}$ is the canonical momentum for the $i$ th particle in the system. $\vec{A}(\vec{r})=-(1 / 2)(\vec{r} \times \vec{B})$ is the vector potential corresponding to a uniform, time-dependent magnetic field, $\vec{E}(t)$ is a time-dependent uniform electric field, and $V(\vec{r})$ is the interaction potential between particles. The last term represents an external harmonic potential acting on the particles.

The physics of the exact mapping can be seen by performing a coordinate transformation to the center of mass (CM) frame of the many-particle system. In the nonrelativistic limit, the wave functions in the laboratory and CM frames are related by

$$
\psi_{l a b}\left(\vec{r}_{i} ; t\right)=\psi_{C M}\left(\vec{r}_{i}-\vec{R}(t) ; t\right) e^{i \theta[\vec{R}(t)]},
$$

where $\vec{R}(t)=(1 / N) \sum_{i}\left\langle\vec{r}_{i}(t)\right\rangle$ is the center of mass coordinate in the laboratory frame, $N=$ number of particles, and $\theta[\vec{R}(t)]$ is an overall phase that depends on $\vec{R}(t)$ only. The corresponding Hamiltonian in the CM frame is 


$$
\begin{aligned}
H^{\prime}(t)= & \sum_{i} \frac{1}{2 m}\left(\vec{p}_{i}^{\prime}-m \dot{\vec{R}}(t)-\frac{e}{c} \vec{A}_{C M}^{\prime}\left(\vec{r}_{i}^{\prime}\right)\right)^{2}+\frac{1}{2} \sum_{i \neq j} V\left(\vec{r}_{i}^{\prime}-\vec{r}_{j}^{\prime}\right) \\
& +\frac{1}{2} \sum_{i}\left[\vec{r}_{i}^{\prime}+\vec{R}(t)\right] \cdot \stackrel{\leftrightarrow}{K} \cdot\left[\vec{r}_{i}^{\prime}+\vec{R}(t)\right]-e \sum_{i} \vec{r}_{i}^{\prime} \cdot \vec{E}^{\prime}(t),
\end{aligned}
$$

where $\quad \vec{r}^{\prime}=\vec{r}-\vec{R}(t), \quad \vec{p}_{i}^{\prime}=-i \bar{h} \nabla^{\prime}, \quad$ and $\quad \vec{A}^{\prime}\left(\vec{r}^{\prime}\right)=-(1 / 2) \vec{r}^{\prime}$ $\times \vec{B}^{\prime} \cdot \vec{B}^{\prime}=\vec{B}$ and $\vec{E}^{\prime}(t)=\vec{E}(t)+(1 / c) \dot{\vec{R}}(t) \times \vec{B}$ are the Galilean transformed magnetic and electric fields in the CM frame valid in the nonrelativistic limit. The Schrödinger equation in the CM frame, $i \hbar \dot{\psi}_{C M}=H^{\prime}(t) \psi_{C M}$ can be simplified by a performing a gauge transformation $\psi_{C M}=\phi_{C M} e^{i \Lambda(t)}$, where $\hbar \Lambda(t)=\Sigma_{i}\left[m \dot{\vec{R}}(t) \cdot \vec{r}^{\prime}-\frac{1}{2} \int^{t} d t^{\prime} \vec{R}\left(t^{\prime}\right) \cdot \overleftrightarrow{K} \vec{R}\left(t^{\prime}\right)\right]$. With Eq. (4), we obtain

$$
i \hbar \frac{\partial}{\partial t} \phi_{C M}\left(\vec{r}^{\prime} ; t\right)=\left(H_{o}\left(\vec{r}_{i}^{\prime}\right)-\sum_{i} \vec{r}_{i}^{\prime} \cdot \vec{a}(t)\right) \phi_{C M}\left(\vec{r}^{\prime} ; t\right),
$$

where $\quad \vec{a}(t)=e\{\vec{E}(t)+(1 / c)[\dot{\vec{R}}(t) \times \vec{B}]\}-\overleftrightarrow{K} \cdot \vec{R}(t)-m \ddot{\vec{R}}(t)=0$. $\vec{a}(t)$ vanishes because it is the equation of motion for $\vec{R}(t)$ which can be derived directly from the corresponding Heisenberg equation of motion. We thus arrive at the conclusion that for the class of Hamiltonian (2), there exist a oneto-one mapping between the solution of the Schrödinger equation in the presence of the microwave radiation $\psi\left(\vec{r}_{i} ; t\right)$, and in the absence of the radiation $\phi_{C M}\left(\vec{r}_{i}^{\prime} ; t\right)$, where $\psi\left(\vec{r}_{i} ; t\right)=\phi_{C M}\left(\vec{r}_{i}-\vec{R}(t) ; t\right) e^{i \Lambda(t)}$. Physically, for the particular form of Hamiltonian we considered, the wave function of the system follows the center of mass motion rigidity in the presence of the radiation field.

The above result suggests that for more general Hamiltonians of form $H_{G}(t)=H(t)+U$, a perturbation scheme where the microwave radiation is treated exactly to all order can be set up by treating $U$ as perturbation. The perturbation scheme can be set up most easily in the center of mass frame. We shall consider static impurity potential $U=\lambda \Sigma_{i} u\left(\vec{r}_{i}\right)$ in the following. Notice that a static potential becomes timedependent in the CM frame and should be treated by timedependent perturbation theory.

To derive the transport equation we start from the exact Heisenberg equation of motion for the center of mass coordinate, $i \hbar \dot{\vec{R}}(t)=\left[\vec{R}(t), H_{G}(t)\right]$ with $\overleftrightarrow{K}=0^{4}$. We obtain after some simple algebra

$$
m \ddot{\vec{R}}(t)=e\left(\vec{E}(t)+\frac{1}{c}[\dot{\vec{R}}(t) \times \vec{B}]\right)-\frac{\lambda}{N} \int d^{d} r[\nabla u(\vec{r})] n(\vec{r}, t),
$$

where $n(\vec{r}, t)=\sum_{i}\left\langle\delta\left(\vec{r}-\vec{r}_{i}\right)\right\rangle$ is the time-dependent average electron density. In the CM frame where $n(\vec{r}, t)=n_{C M}[\vec{r}$ $-\vec{R}(t), t]$, we obtain for small $\lambda$ from linear response theory

$$
\begin{aligned}
n_{C M}\left(\vec{r}^{\prime}, t\right)= & n_{C M}^{(0)}\left(\vec{r}^{\prime}\right)+\lambda \int d^{d} r^{\prime \prime} d t^{\prime \prime} \chi\left(\vec{r}^{\prime}-\vec{r}^{\prime} ; t-t^{\prime}\right) \\
& \times u\left[\vec{r}^{\prime \prime}+\vec{R}\left(t^{\prime}\right)\right],
\end{aligned}
$$

where $\chi\left(\vec{r}^{\prime}-\vec{r}^{\prime \prime} ; t-t^{\prime}\right)$ is the (equilibrium) retarded densitydensity response function in CM frame given by the Hamiltonian $H_{o}$. Going back to the laboratory frame and performing the disorder-average $\langle u(\vec{r})\rangle=0$ and $\left\langle u(\vec{r}) u\left(\vec{r}^{\prime}\right)\right\rangle=|u|^{2} \delta(\vec{r}$ $\left.-\vec{r}^{\prime}\right)$, we obtain to second order in $\lambda$, an impurity-averaged equation of motion for $\vec{R}(t)$ in laboratory frame, ${ }^{4}$

$$
\begin{aligned}
m \ddot{\vec{R}}(t)= & e\left(\vec{E}(t)+\frac{1}{c}[\dot{\vec{R}}(t) \times \vec{B}]\right)+\alpha \nabla_{\vec{R}(t)} \\
& \times \int d t^{\prime} \chi\left[\vec{R}(t)-\vec{R}\left(t^{\prime}\right) ; t-t^{\prime}\right],
\end{aligned}
$$

where $\bar{n}=N / V$ and $\alpha=\lambda^{2}|u|^{2} / \bar{n}$. The equation is manifestly gauge invariant and suggests that to second order in the impurity potential, the effects of particle statistics and interaction are reflected only in the density-density response function. In the following we shall apply this equation to study the ZRS in two-dimensional (2D) electron systems. We note that the same equation has been obtained by Lei et al., ${ }^{4}$ where they consider electric fields of form $\vec{E}(t)=\vec{E}_{1} \cos (\omega t)$ $+\vec{E}_{2} \sin (\omega t)+\vec{E}_{d}$, where the first two terms represent microwave radiation with frequency $\omega$ and $\vec{E}_{d}$ is a small dc electric field. To proceed further they consider approximate solutions of form

$$
\dot{\vec{R}}(t)=\vec{v} t+\vec{A} \cos (\omega t)+\vec{B} \sin (\omega t),
$$

where $\vec{v}, \vec{A}, \vec{B}$ are determined self-consistently from Eq. (7).

We proceed differently by first dividing the center of mass motion into "fast" and "slow" parts,

$$
\vec{R}(t)=\vec{R}_{f}(t)+\vec{R}_{s}(t) \sim \vec{A}_{o} \cos (\omega t)+\vec{B}_{o} \sin (\omega t)+\vec{R}_{s}(t),
$$

where $\vec{R}_{f}(t)$ describes the center of mass motion induced by the radiation field whereas $\vec{R}_{s}(T)$ describes the rest of the motion either spontaneously generated or induced by $\vec{E}_{d}$. We shall eliminate $\vec{R}_{f}(t)$ to obtain an explicit equation of motion for $\vec{R}_{s}(t)$. The two kinds of motions are coupled by the impurity scattering term which is a nonlinear function of $\vec{R}(t)$. We shall assume that the coupling between $\vec{R}_{s}(t)$ and $\vec{R}_{f}(t)$ does not modify qualitatively the behavior of $\vec{R}_{f}(t)$ and the main effect of coupling is to produce an effective equation of motion for $\vec{R}_{s}(t)$. Notice that we keep only the first harmonic terms in $\vec{R}_{f}(t)$ in Eq. (8). This is valid in the small $\vec{E}_{o}$ limit when the size of the orbit $R_{c} \sim \sqrt{\vec{A}_{o}^{2}+\vec{B}_{o}^{2}}$ is much less than the magnetic length $l=(\hbar c / e B)^{1 / 2}$ and is justifiable under the experimental condition, ${ }^{1,2}$ where the magnetic field is weak and the magnetic length is very long.

To treat the impurity scattering term we write 


$$
\nabla_{\vec{R}(t)} \int_{-\infty}^{t} d t^{\prime} \chi\left(\vec{R}(t)-\vec{R}\left(t^{\prime}\right) ; t-t^{\prime}\right)=-\int \vec{q} d^{d} q \int\left(d \omega^{\prime} / \pi\right) \operatorname{Im} \chi\left(\vec{q}, \omega^{\prime}\right) \int_{-\infty}^{t} d t^{\prime} e^{\left.i l \vec{q} \cdot\left[\vec{R}(t)-\vec{R}\left(t^{\prime}\right)\right]-\omega^{\prime}\left(t-t^{\prime}\right)\right\}},
$$

where $\chi(\vec{q}, \omega)$ is the Fourier transform of $\chi(\vec{r} ; t)$. To evaluate the integral over $t^{\prime}$ we make the local approximation $\vec{R}_{s}(t)$ $-\vec{R}_{s}\left(t^{\prime}\right) \sim \vec{v}(t)\left(t-t^{\prime}\right)+(1 / 2) \dot{\vec{v}}(t)\left(t-t^{\prime}\right)^{2}$, and

$$
\int_{-\infty}^{t} d t^{\prime} e^{i\left\{\vec{q} \cdot\left[\vec{R}(t)-\vec{R}\left(t^{\prime}\right)\right]-\omega^{\prime}\left(t-t^{\prime}\right)\right\}} \sim \int_{-\infty}^{t} d t^{\prime} e^{i\left\{\vec{q} \cdot\left[\vec{R}_{f}(t)-\vec{R}_{f}\left(t^{\prime}\right)\right]+\left[\vec{q} \cdot \vec{v}(t)-\omega^{\prime}\right]\left(t-t^{\prime}\right)\right\}}\left(1+i \vec{q} \cdot \dot{\vec{v}}(t) \frac{\left(t-t^{\prime}\right)^{2}}{2}\right)
$$

valid for $\vec{v}\left(t+T^{\prime}\right)-\vec{v}(t) \ll \vec{v}(t)$, where $T^{\prime} \sim 2 \pi / \omega_{B}$ is the characteristic time scale for the density response. The $\vec{R}_{f}(t)$ $-\vec{R}_{f}\left(t^{\prime}\right)$ term can be expanded in a series of $\vec{A}_{o}, \vec{B}_{o}$ using the identity $e^{i x \sin \theta}=\Sigma_{m} J_{m}(x) e^{i m \theta}$. We obtain after some algebra,

$$
\begin{aligned}
\nabla_{\vec{R}(t)} \int_{-\infty}^{t} d t^{\prime} \chi\left(\vec{R}(t)-\vec{R}\left(t^{\prime}\right) ; t-t^{\prime}\right) \\
=\sum_{m, m^{\prime}} e^{i\left(m-m^{\prime}\right)(\omega t+\theta)} \int \vec{q} d^{d} q J_{m}[z(\vec{q})] J_{m^{\prime}}[z(\vec{q})] \\
\quad \times\left(1-i \frac{\vec{q} \cdot \dot{\vec{v}}(t)}{2} \frac{\partial^{2}}{\partial \omega^{\prime 2}}\right) \chi\left(\vec{q}, \omega^{\prime}\right)_{\omega^{\prime}=m \omega+\vec{q} \cdot \vec{v}(t)},
\end{aligned}
$$

where $z(\vec{q})=\sqrt{\left(\vec{q} \cdot \vec{A}_{o}\right)^{2}+\left(\vec{q} \cdot \vec{B}_{o}\right)^{2}}$ and $\tan \theta=\vec{q} \cdot \vec{A}_{o} / \vec{q} \cdot \vec{B}_{o} \cdot{ }^{4}$ We shall consider $\vec{E}_{2}= \pm \hat{z} \times \vec{E}_{1}$, corresponding to circularly polarized light. Neglecting the influence of $\vec{R}_{s}(t)$ on $\vec{R}_{f}(t)$ we obtain $\vec{B}_{o}= \pm \hat{z} \times \vec{A}_{o}$, i.e., circular orbits and $z(\vec{q})^{2}=R_{c}^{2} q^{2} / 2$.

The effective force on $\vec{R}_{s}(t)$ is derived from the $m=m^{\prime}$ terms in (9). To order $\left(R_{c} / l\right)^{2}$ we keep only $m\left(m^{\prime}\right)=0,1$ terms. Putting it back into the equation of motion (7), we obtain

$$
\{m+\Pi[\omega, v(t)]\} \dot{\vec{v}}(t)=e \vec{E}_{d}+\frac{e}{c}[\vec{v}(t) \times \vec{B}]-R[\omega, v(t)] \vec{v}(t),
$$

where $\omega$ is the frequency of the microwave radiation, $v(t)$ $=|\vec{v}(t)|$, and

$$
\begin{gathered}
R[\omega, v] \sim \frac{\alpha}{v^{2}} \int \vec{q} \cdot \vec{v} d^{d} q\left[\left(1-\frac{z(\vec{q})^{2}}{2}\right) \operatorname{Im} \chi(\vec{q}, \vec{q} \cdot \vec{v})\right. \\
\left.+\frac{z(\vec{q})^{2}}{4} \operatorname{Im} \chi(\vec{q}, \omega+\vec{q} \cdot \vec{v})\right], \\
\Pi[\omega, v] \sim-\frac{\alpha}{4} \int q^{2} d^{d} q \frac{\partial^{2}}{\partial \omega^{\prime 2}}\left[\left(1-\frac{z(\vec{q})^{2}}{2}\right) \operatorname{Re} \chi\left(\vec{q}, \omega^{\prime}\right)_{\omega^{\prime}=\vec{q} \cdot \vec{v}}\right. \\
\left.+\frac{z(\vec{q})^{2}}{4} \operatorname{Re} \chi\left(\vec{q}, \omega^{\prime}\right)_{\omega^{\prime}=\omega+\vec{q} \cdot \vec{v}}\right] .
\end{gathered}
$$

It is obvious that $R[\omega, v]$ represents dissipative response of the electron gas to external perturbations whereas $\Pi[\omega, v]$ is an effective mass correction on the center of mass motion coming from the corresponding reactive response. Negative contributions to the resistance shows up in the second term of $R$ for density-density response functions with resonant structure, $\chi(\vec{q}, \omega) \sim \sum_{n}\left[g_{n}(\vec{q}) / \pi\right]\left[-2 \omega_{n} /\left(\omega^{2}-\omega_{n}^{2}\right)\right]$, where $\omega_{n}$ $>0$ are resonant energy levels and $g_{n}(\vec{q})$ is a positive definite function. In this case, $R \sim \sum_{n}\left[R_{1 n} \omega_{n}+R_{2 n}\left(\omega_{n}-\omega\right)\right]$, where $R_{1 n}$ and $R_{2 n}$ are positive definite numbers. We see that negative contributions to $R_{2}$ exist for $\omega_{n}<\omega$. The physical origin of the negative resistance has been discussed in several earlier works $3,4,6,7,12$ and we shall not repeat them here. Correspondingly, the effective mass contribution from level $n$ is positive (negative) when $\omega>(<) \omega_{n}$. We note that the effective mass correction is of order $\Pi[\omega, v] \sim m \times\left(1 / \omega_{B} \tau\right) \ll m$ in the weak-disorder limit, where $\omega_{B}=e M / m c$ is the cyclotron frequency and $\tau \sim\left(g_{2 d} \lambda^{2}|u|^{2}\right)^{-1}$ is the elastic lifetime. Equation (10) differs from the phenomenological Eq. (1) mainly in the presence of the inertial term $m \dot{\vec{v}}$ which allows the system to admit time-dependent solutions in the present case.

\section{ANALYSIS OF THE TRANSPORT EQUATION}

To see whether $R[\omega, v]$ has the expected behavior we consider the density response function of noninteracting gases where ${ }^{4}$

$$
\begin{aligned}
\chi(\vec{q}, \omega)= & \left(2 \pi l^{2}\right)^{-1} \sum_{m, n}\left(n_{2} ! / n_{1} !\right)\left(Q^{2} / 2\right)^{n_{1}-n_{2}} e^{-\left(Q^{2} / 2\right)} \\
& \times\left[L_{n_{2}}^{n_{1}-n_{2}}\left(Q^{2} / 2\right)\right]^{2}\left(\frac{n_{F}\left(n \omega_{B}\right)-n_{F}\left(m \omega_{B}\right)}{\omega+(n-m) \omega_{B}+i \delta}\right),
\end{aligned}
$$

where $n_{1}=\max (n, m), n_{2}=\min (n, m), L_{m}^{n}(x)$ is the associated Laguerre polynomials, $n_{F}(\epsilon)$ is the Fermi distribution function, and $Q^{2}=|\vec{q}|^{2} l^{2}$. To incorporate inelastic lifetime effects we also introduce a phenomenological broadening $\Gamma(T)$ to the Landau levels, i.e., $\delta\left(\epsilon-n \omega_{B}\right) \rightarrow(\pi)^{-1} \Gamma /\left[\left(\epsilon-n \omega_{B}\right)^{2}+\Gamma^{2}\right]$. $R[\omega, v]$ is evaluated numerically with these approximations. In Fig. 1 we present numerical results for the normalized resistance $R(\omega, v) / R(0,0)$, as a function of normalized velocity $v_{N}=v /\left(l \omega_{B}\right)$ for $\omega / \omega_{B}=0,0.85,1.0,1.1,1.2$, taking $\left(R_{c} / l\right)^{2}=0.1, E_{F} \sim 10 \omega_{B}, T=2 \omega_{B}, \Gamma=0.5$, and keeping 20 levels in the sum. We observe that $R(\omega, v \rightarrow 0)$ is a function of frequency and becomes negative when $\omega \gtrsim \omega_{B}$, in agreement 


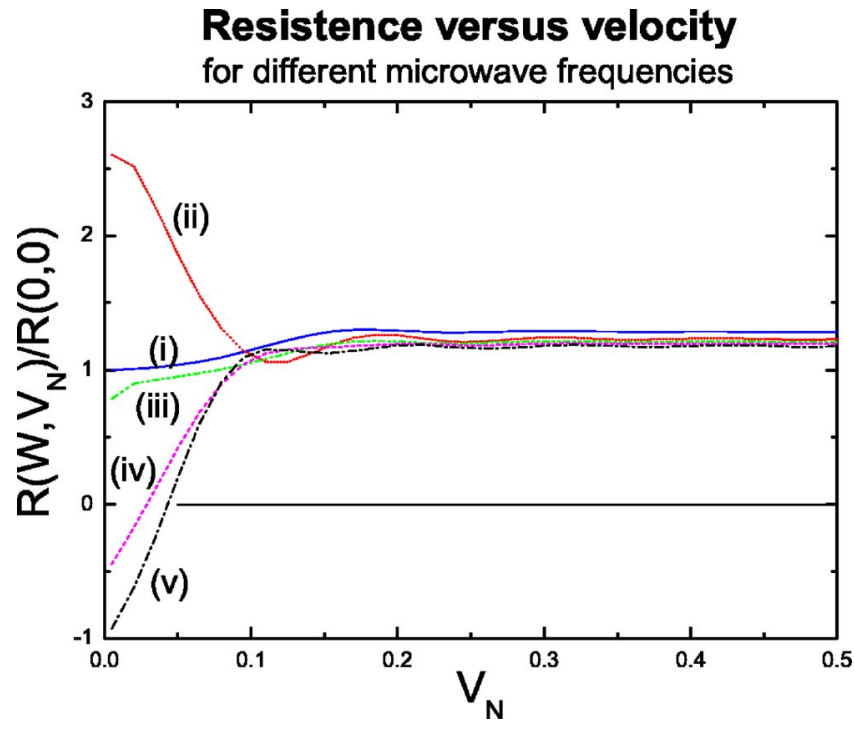

FIG. 1. (Color online) Normalized resistance as a function of velocity for $\omega / \omega_{B}=0$ (i) 0.85 (ii), 1.0 (iii), 1.1 (iv), and 1.2 (v).

with previous results. ${ }^{12}$ For $\omega>\omega_{B} R(\omega, v)$ 's increases and cross zero at around $v_{o N} \sim 0.05$. The effect of microwave radiation decreases rapidly for $v_{N} \geq 0.2$. These qualitative behaviors of $R(\omega, v)$ are in agreement with our expectation and are not modified by changing $T$ or $\Gamma$. We note that the same conclusion was also obtained by $\mathrm{Lei}^{5}$ in an earlier numerical analysis.

Equation (10) allows time-dependent solutions. In the absence of the dc field, a simple, spatially homogeneous solution which allows the system to stabilize itself around the point $v_{o}=\left(l \omega_{B}\right) v_{o N}$ is

$$
\vec{v}^{(0)}(t)=R_{o}\left[\cos \left(\omega_{s} t\right) \hat{x}+\sin \left(\omega_{s} t\right) \hat{y}\right],
$$

with $R_{o}=v_{o} / \omega_{s}$, where $\omega_{s}=e B /\left[m+\Pi\left(\omega, v_{o}\right)\right] c \sim \omega_{B}$. The solution represents a collective circular motion of the whole fluid moving with speed $v_{o}$. Notice that a time-independent, spatially inhomogeneous solution corresponding to a pattern of alternating current stripes ${ }^{9}$ may still exist. However, this solution is energetically less favorable because it requires a higher energy to create the charge inhomogeneity needed to maintain the stripes of currents.

We shall now argue that a ZRS can also be generated from the above solution when we consider the boundaries of the droplet of electron fluid. For sharp boundaries the boundary condition $j_{\perp}=0$ has to be imposed where $j_{\perp}$ is the component of current perpendicular to the boundary. As a result an edge region with a time-independent current $j_{\|} \sim j_{o}$ must form. The size of this region is determined by the microscopic charge dynamics ${ }^{7}$ which is still undetermined. Nevertheless according to Eq. (10) an electric field perpendicular to the boundary with magnitude $\sim E_{d}=B v_{o} / c$ has to be present in this region to maintain the steady current flow. A similar edge region also exists at the opposite edge with a current running in the opposite direction, rather similar to edge states in quantum Hall effect.

Similar to the "stripe" solution a state with a small net current flow can be created with minimal disturbance to the system by shrinking the size of one edge region and enlarging the other. In this case, the net voltage drop across the sample is given by

$$
V_{y}=\int E(y) d y=\frac{B}{c} \int v_{x}(y) d y=\frac{B}{n e^{2} c} \int j_{x}(y) d y=\rho_{H} I_{x},
$$

corresponding to a resistance matrix with $\rho_{x y}=\rho_{H}$, i.e., the ZRS.

\section{COMMENTS AND CONCLUSION}

Some comments about the validity of our theory is in order. We note that the oscillatory solution is allowed because of the presence of the inertia term $m \dot{\vec{v}}(t)$. In usual transports with finite dissipation, a steady dc solution exists and this term is irrelevant and can be neglected. However, this term becomes important when dissipation vanishes and a steady dc solution becomes unstable. Our analysis shows that the correction to the inertia term is small $\left[\sim m /\left(\omega_{B} \tau\right)\right]$ in the limit of weak disorder and the local approximation we made in deriving the equation of motion mainly affects $R[\omega, v]$. In particular, our general description of the ZRS should remain valid as long as the qualitative property of $R[\omega, v]$ is correct. Another simplification we employed in our analysis is the assumption of isotropic responses. The response functions $\Pi[\omega, v]$ and $R[\omega, v]$ become anisotropic tensors for planepolarized light $t^{5,12}$ and for circularly polarized light when the effect of $\vec{R}_{s}(t)$ on $\vec{R}_{f}(t)$ are included. The effect of anisotropy in $\Pi$ and $R$ can be studied phenomenologically by adding anisotropy terms in the equation of motion. For example, for plane-polarized light, we may consider

$$
\begin{gathered}
(m+\delta m) \dot{v}_{x}(t) \hat{x}+(m-\delta m) \dot{v}_{y}(t) \hat{y}=e \vec{E}_{d}+\frac{e}{c}[\vec{v}(t) \times \vec{B}] \\
-[R(v)+\delta r] v_{x}(t) \hat{x}-[R(v)-\delta r] v_{y}(t) \hat{y},
\end{gathered}
$$

where we assume the microwave electric field to be along the $\hat{x}$-axis and $R(v) \sim R\left(v^{2}-v_{o}^{2}\right)$. For small $\delta m$ and $\delta r$ the effect of anisotropic terms can be analyzed in perturbation theory. A linear stability analysis around the circular orbit (12) indicates that in the limit $\omega_{B} \tau \gg 1$ these terms do not introduce any singular perturbation and the circular orbit is only perturbed slightly.

Lastly we made a comment on the macroscopic nature of the spontaneous current state we proposed. We note that in general a spontaneous current state with $|\vec{v}(t)|=v_{o}$ is characterized by a position and time dependent (2D) unit vector field $\hat{n}(\vec{x}, t)$ representing the direction of the current. The order parameter field $\hat{n}(\vec{x}, t)$ has the same symmetry as the ordinary 2D $x-y$ model, or superfluids. The main difference between the ZRS state and superfluids is that the rigidity of the order parameter is protected by repulsive interaction in 
the case of superfluids, whereas it is protected by the principle of least dissipation in the ZRS. The similarity between the two systems suggests that the two systems may share some common macroscopic features. For example, vortexlike solitonic excitations may exist in the ZRS and may lead to the residue resistance $\sim R_{o} \exp \left[-\left(T_{o} / T\right)\right]$ observed in the ZRS state. ${ }^{1,2}$ The existence and nature of solitonic excitations

${ }^{1}$ R. G. Mani, J. H. Smet, K. von Klitzing, V. Narayanamurti, W. B. Johnson, and V. Umansky, Nature 420, 646 (2002).

${ }^{2}$ M. A. Zudov, R. R. Du, L. N. Pfeiffer, and K. W. West, Phys. Rev. Lett. 90, 046807 (2003).

${ }^{3}$ A. C. Durst, S. Sachdev, N. Read, and S. M. Girvin, Phys. Rev. Lett. 91, 086803 (2003).

${ }^{4}$ X. L. Lei and S. Y. Liu, Phys. Rev. Lett. 91, 226805 (2003); X. L. Lei, J. Appl. Phys. 84, 1396 (1998); X. L. Lei and S. Y. Liu, Eur. J. Phys. B13, 271 (2000).

${ }^{5}$ X. L. Lei, J. Phys.: Condens. Matter 16, 4045 (2004).

${ }^{6}$ Junren Shi and X. C. Xie, Phys. Rev. Lett. 91, 086801 (2003).

${ }^{7}$ A. V. Andreev, I. L. Aleiner, and A. J. Millis, Phys. Rev. Lett. 91, depends on the detailed charge and current dynamics of the ZRS state and will be investigated in a coming paper.

\section{ACKNOWLEDGMENTS}

This work is supported by the HKRGC through Grant No. 602803.

056803 (2003)

${ }^{8}$ K. Park, Phys. Rev. B 69, 201301(R) (2004).

${ }^{9}$ J. Iñarrea and G. Platero, Phys. Rev. Lett. 94, 016806 (2005).

${ }^{10}$ C. L. Yang, M. A. Zudov, T. A. Knuuttila, R. R. Du, L. N. Pfeiffer, and K. W. West, Phys. Rev. Lett. 91, 096803 (2003).

${ }^{11}$ J. Zhang, S. K. Lyo, R. R. Du, J. A. Simmons, and J. L. Reno, Phys. Rev. Lett. 92, 156802 (2004).

${ }^{12}$ M. G. Vavilov and I. L. Aleiner, Phys. Rev. B 69, 035303 (2004); I. A. Dmitriev, M. G. Vavilov, I. L. Aleiner, A. D. Mirlin, and D. G. Polyakov, ibid. 71, 115316 (2005).

${ }^{13}$ J. F. Dobson, Phys. Rev. Lett. 73, 2244 (1994). 